\title{
Quantum Information and Quantum Black Holes
}

\author{
Jacob D. Bekenstein \\ The Racah Institute of Physics, Hebrew University of Jerusalem \\ Givat Ram, Jerusalem 91904, Israel
}

\begin{abstract}
First, the relation between black holes and limitations on information of other systems is developed. After reviewing the relation of entropy to information, we derive the entropy bound, review its applications to cosmology and its extensions to higher dimensions, and discuss why black holes behave as 1-D objects when emitting entropy. We also discuss fundamental limitations on the information of pulses in curved space, and on the rate of disposal of information into a black hole. We then move on to a discussion of quantum black holes motivated by the adiabatic invariance of horizon area of classical holes. We develop an algebraic formalism based on symmetry which gives information on the area (or mass) spectrum of quantum black holes, and on the degeneracy of the levels. This last turns out to be consistent with the proportionality of black hole entropy and horizon area, while leaving room for corrections.
\end{abstract}

\section{Limitations on Quantum Information from Black Holes}

\section{$1.1 \quad$ Introduction}

A naive view of physics-classical or quantum - would have us believe that an infinite amount of information can be contained in a finite 3-D space. After all, matter can have an infinity of classical configurations there, and quantum fields have an infinity of modes in that region. 't Hooft and Susskind's holographic principle [1, 2] has shattered this popular view: it holds that insofar as the information required to describe them is concerned, physical systems are inherently two-dimensional in space. In particular, the information or entropy (see Sec. 1.2 below for the relation) in an isolated system is expected to be bounded from above by one quarter the area of a circumscribing surface expressed in Planck units (holographic bound):

$$
S \leq A(4 \hbar)^{-1}
$$

Except as otherwise stated, I assume units with $G=c=1$. 't Hooft's original statement of the holographic principle [1] was elucidated by Susskind [2] who showed that the holographic bound is required by the generalized second law (GSL) [3] applied

\footnotetext{
${ }^{1}$ email: bekenste@vms.huji.ac.il
} 
to the wholesale collapse of a physical system into a black hole of its own making. A loophole in Susskind's argument pointed out by Wald [4] can be corrected for quiescent systems by considering either infall of the system into a large black hole, or a tiny auxiliary black hole which devours the system [5]. The holographic bound as above stated can be exceeded by dynamically evolving systems, but Bousso [6] has given a reformulation which works in these cases also.

The attention of the particle theory community has been riveted on the implications of the holographic principle for the structure of the fundamental equations of nature, for example the equivalence between physical theories formulated in different spacetime dimensions. However, there is no gainsaying the possible practical importance of fundamental restrictions, such as the holographic one on the information storage capacity of systems. For one thing, the principles obviously serve as a final arbitrates of the promise of any futuristic information storage technology. In this respect the holographic bound is not an onerous one. For instance, it merely requires that a device with dimensions of order a centimeter hold no more than some $10^{66}$ bits of information. By contrast, all the books in the Library of Congress hold of the order of $10^{15}$ bits of information, and no state-of-the-art one-centimeter size memory can hold all that. The holographic bound is thus too liberal for the present and foreseeable technology. So one question to be asked is, can one device a tighter bound on information storage than the holographic one? In light of the explosive development of fast communications, a further interesting question would be: what fundamental bounds can be set on the flow of information? In this lecture I shall go into such questions wherever they infringe into the realm of gravity, which I here take to be described by standard general relativity.

\subsection{Information and entropy}

It is plain that information can be stored in a system, by man or by nature, only if that system has more than one state accessible for the task. Nothing can be learned from a system that looks the same under all circumstances. With two distinguishable states with no bias between them, one can already store and retrieve information, for example, the answer to a "yes or no" question. A two-state system can thus hold a bit of information. What about a $N$ state system with no bias between states ? It makes sense to assign it information capacity $\log _{2} N$ bits. For one thing, this reduces to the previous case for $N=2$. In addition, if we have two well separated classical systems, $\mathrm{A}$ and $\mathrm{B}$, with $N_{\mathrm{A}}$ and $N_{\mathrm{B}}$ (unbiased) states, respectively, there are a total of $N_{\mathrm{A}} N_{\mathrm{B}}$ overall states, so we would assign the joint system information capacity $\log _{2}\left(N_{\mathrm{A}} N_{\mathrm{B}}\right)$. But this is just the sum of information capacities for $\mathrm{A}$ and for B; thus is the logarithm function singled out as the relevant one for quantifying information. The states mentioned must be precisely distinguishable. In classical physics they may be, for example, mutually nonoverlapping cells in the phase space of a multiparticle system. In quantum theory they must be mutually orthogonal states, because nonorthogonal states cannot be distinguished with certainty by any 
measurement [7].

What if the states are biased ? For instance, it might be that before measurement is made, state 1 is twice as probable as state 2 . Does this affect the information capacity? Let us imagine that our system has $N$ equally probable states, but we divide these in groupings of $N_{1}, N_{2}, \cdots$ states with $\sum_{i} N_{i}=N$. If our experimental resolution does not permit us to peer into the groupings (or we just do not care to do so), we have to consider them as regular states with probabilities $p_{1}=N_{1} / N, p_{2}=N_{2} / N, \cdots$. It is reasonable to expect that the information capacity of the system, call it $I_{\max }$, should depend only on the set $\left\{p_{i}\right\}: I_{\max }=I_{\max }\left(p_{1}, p_{2}, \cdots\right)$. However, had we insisted and succeeded in peering into the groupings, we could have obtained additional information $\log _{2} N_{1}$ from the first grouping (which turns up with probability $p_{1}$ ), and so on. Therefore, the total information capacity of the system can be written in two ways which must agree:

$$
I_{\max }\left(p_{1}, p_{2}, \cdots\right)+\sum_{i} p_{i} \log _{2} N_{i}=\log _{2} N
$$

Using the normalization $\sum_{i} p_{i}=1$ it is easy to solve for $I_{\max }$ :

$$
I_{\max }\left(p_{1}, p_{2}, \cdots\right)=-\sum_{i} p_{i} \log _{2} p_{i}
$$

This is Shannon's famous 1948 formula 8 for the peak information capacity of a system with distinguishable states which occur with a priori probabilities $\left\{p_{i}\right\}$. It clearly makes no difference for the final result whether the states $\{i\}$ are composite or "elementary", so long as the probabilities $p_{i}$ assigned them reflect an operational expectation.

Shannon's $I_{\max }$ looks like Boltzmann's expression for the entropy of a gas, apart from the facts that Boltzmann used natural logarithms, and that he prefaced the expression with the constant $k$, an historical accident stemming from the use of different units for temperature and energy. Beneath these superficialities the two are the same expression: thermodynamic entropy is the information storage capacity of matter. And thermodynamics is derivable from information-theoretic concepts [9, 77. The endless controversies in the literature around this information-entropy equivalence seem to stem from confusion about the level at which the probabilities $\left\{p_{i}\right\}$ are formulated. The chemist, for instance, determines the entropy $S$ of a piece of iron by methods that reach down to the atomic level; for him the states $\{i\}$ are atomic states. The engineer, by contrast, is interested in storing information in the magnetic domains of the iron in a magnetic tape. He groups atomic states into domain states which give him new probabilities $p_{i}{ }^{\prime}$. The $I_{\max }$ he calculates from these is much smaller than the chemist's $S$, but there is no question of conceptual contradiction.

Likewise, confusion has arisen about the relation between von Neumann's entropy in quantum theory, $S=-\operatorname{Tr} \rho \ln \rho$ ( $\rho$ is the density matrix), and Shannon's $I_{\max }$. Apart from an obvious factor $\ln 2$, the former is identical to $I_{\max }$ when the $p_{i}$ 's in the latter refer to the probabilities to measure values of an observable whose eigenstates 
coincide with those of $\rho$ (for example energy when $\rho$ stands for a thermal state). But it is possible to design quantum measurements for which the probabilities will give a Shannon $I_{\max }$ which exceeds von Neumann's $S$. In this case the states are no longer mutually orthogonal, and so are not fully distinguishable, and the maximum information that can be read out of the system is no longer equal to (3), but is bounded by $S \log _{2} e$ [7]. At any rate, there is never a question of $I_{\max }$ and $S$ being unrelated.

The chemist and engineer in the previous discussion are concerned with two levels of probabilities. In reality there are many: matter is made of atoms which are themselves built of electrons and nuclei. The last are put together from nucleons which are themselves composites of various quarks and gluons. There are states, and so probabilities and an entropy, at every level of this hierarchy. Obviously, the deeper we go, the higher the entropy. In what follows we shall be interested in the entropy (information capacity) $S_{\mathrm{X}}$ calculated at level X, the deepest level of structure. For ordinary matter this means the level of lepton, quark and gluon degrees of freedom, or something deeper if the standard model is up for a big revision. Obviously this $S_{\mathrm{X}}$ bounds from above the information capacity of material media accessible with any actual technology.

\subsection{The entropy bound}

Consider in asymptotically flat four-dimensional spacetime $(D=n+1=4)$ a finite system $\mathcal{U}$ with energy $E$ which, for the moment, we think of as spherical with radius $R$ ( $E$ and $R$ measured in proper frame). How big can its entropy $S$ be ? The holographic principle limits it to $S \leq 4 \pi R^{2} / \hbar$ (this is in natural logarithm units, nits, rather than base-two logarithm units, bits). Can we do better without knowing more details about $\mathcal{U}$ ? Indeed, we have the entropy bound

$$
S \leq 2 \pi E R / \hbar
$$

proposed prior to the advent of holography [10]. So long as $\mathcal{U}$ 's self-gravity is not strong, $E \ll R$. For example, for laboratory sized systems $E<10^{-23} R$, while for astronomical systems, barring neutron stars and black holes, $E<10^{-5} R$. Thus, with few exceptions, the entropy bound is many orders of magnitude tighter than the holographic one. It restricts the information capacity of a one-centimeter device made of ordinary matter to be less than $10^{37}$ bits, which limit no longer looks unreachable.

In the original derivation of bound (4) I imagined that $\mathcal{U}$ is lowered slowly from far away to the horizon of a stationary black hole, while all the freed potential energy is allowed to do work on a distant agent (a Geroch process [11]). I then applied the GSL to get the bound [10]. This derivation was criticized [12] for not taking into account the buoyancy of $\mathcal{U}$ in the Unruh radiation surrounding it by virtue of its acceleration. A protracted controversy [12, 13] on this issue led to the perception 114 that correction for buoyancy - itself an intricate calculation - merely increases the $2 \pi$ coefficient in Eq. (田) by a tiny amount provided only that one assumes that 
$R \geq \hbar / E$. Now elementary particles and composite objects do obey this restriction. (Some solitons in $D=1+1$ dimensional theories fail to so, but solitons are not expected to bear any entropy anyway.) Although originally meant only for weakly gravitating systems, bound (田) is actually saturated by all Kerr-Newman black holes 110, 15, 16, 17] provided one interprets $E$ as the black hole's mass and $R$ as the Boyer-Lindquist coordinate of the horizon, $r_{+}$.

The entropy in bound (4) is the $S_{\mathrm{X}}$ of Sec. 1.2, and not entropy calculated down to a shallower level of structure. This is because gravitation plays a crucial role in many generic ways of deriving the entropy bound [10, 13, 14, 18]. And gravitation is unique among the interactions in that it is aware of all degrees of freedom in its sources (according to the equivalence principle all energy gravitates). How then could bound (田) take into account only entropy corresponding to intermediate degrees of freedom, and so ignore energy-carrying states at the deeper levels ?

Only of late has the ubiquitous role of bound (4) and closely related ones been realized. For instance, Bousso [19] has shown, via the Geroch process argument, that bound (4) also applies verbatim in any asymptotically flat spacetime of $D=n+1$ dimensions. Further, he shows that when $E$ is expressed in terms of $\mathcal{U}$ 's gravitational radius $r_{\mathrm{g}}$ given by

$$
r_{\mathrm{g}}{ }^{n-2}=\frac{8 \Gamma(n / 2) E}{(n-1) \pi^{n / 2-1}}
$$

the bound takes the form

$$
S \leq \frac{(n-1) \pi^{n / 2} r_{\mathrm{g}}^{n-2} R}{4 \Gamma(n / 2) \hbar^{(n-1) / 2}} .
$$

Bousso has also established a new entropy bound, the D-bound, for systems in $D=n+1$ de Sitter spacetime which occupy a small part of the space inside the cosmological horizon (radius $r_{\mathrm{c}}$ ). It is given again by (6) with $R$ 's role played by $r_{\mathrm{c}}$. Spherical black holes in $D \geq 4$ spacetime (for which the horizon has a $(n-1)$-D "area") also obey (4) in asymptotically flat spacetimes, and the D-bound in asymptotically de Sitter spacetimes, but no longer saturate these bounds for $D>4$ [19].

And somewhat earlier E. Verlinde [20] proposed that the entropy $S$ of a complete closed Robertson-Walker universe in $D=n+1$ spacetime dimensions whose contents are described by a conformal field theory (CFT) - the deeper description of a number of massless fields, possibly in interaction - with large central charge (essentially many particle species), is subject to the generic bound

$$
S \leq \frac{2 \pi R}{n \hbar}\left[E_{\mathrm{C}}\left(2 E-E_{\mathrm{C}}\right)\right]^{1 / 2}
$$

where $R$ is the radius of the $S^{n}$ space, $E$ the total energy in the fields and $E_{\mathrm{C}}$ the Casimir (vacuum) energy. As Verlinde shows, this bound is a straight generalization to $n$ space dimensions of Cardy's famous expression for the entropy of a $D=1+$ 1 CFT [21]. Verlinde points out that for fixed $E$ the maximum of his bound is $2 \pi R E /(n \hbar)$, which never exceeds the original entropy bound (任; indeed Verlinde 
adopts $S \leq 2 \pi R E /(n \hbar)$ as the fiducial form of that bound. A number of recent papers have substantiated Verlinde's bound and so culminate years of protracted efforts by many to make meaningful statements about the entropy (and thus the maximum information) that can be contained in a whole universe 222.

Bounds like (4) are thus of wide applicability. But can one see why a bound like this should be true, at least in some mundane context, without getting embroiled in all the intricacies mentioned ? Indeed, there is an easy gedanken experiment which lets us do this. Drop $\mathcal{U}$ into a Schwarzschild black hole of mass $M \gg E$ from a large distance $d \gg M$ away; $d$ is so chosen that the Hawking radiance carries away energy (as measured at infinity) equal to $E$ while $\mathcal{U}$ is falling to the horizon where it is effectively assimilated by the black hole. At the end of the process the black hole is back at mass $M$ and its entropy has not changed. Were the emission reversible, the radiated entropy would be $E / T_{\mathrm{H}}$ with $T_{H} \equiv \hbar(8 \pi M)^{-1}$. Curved spacetime makes the entropy emitted a factor $\nu$ larger; typical values, depending on particle species, are 23 $\nu=1.35-1.64$. Thus the overall change in world entropy is

$$
\delta S_{\mathrm{ext}}=\delta S_{\mathrm{rad}}-S=\nu E / T_{\mathrm{H}}-S
$$

One can certainly choose $M$ larger than $R$, say, by an order of magnitude so that the system will fall into the hole without being torn up: $M=\zeta R$ with $\zeta=$ a few. Thus by the ordinary second law we obtain the bound

$$
S<8 \pi \nu \zeta R E / \hbar
$$

This bound applies to a rather arbitrary system $\mathcal{U}$ in terms of its total energy $E$ and size $R$. But in the derivation $\mathcal{U}$ is not allowed to be be strongly gravitating (meaning $R \sim E$ ) because then $M$ could not be large compared to $E$, as we have assumed, while $\zeta$ is of order a few. We thus have to assume in addition $R \gg E$.

Note that we could not derive (9) by using a heat reservoir in lieu of a black hole. A reservoir which has gained energy $E$ upon $\mathcal{U}$ 's assimilation, and has returned to its initial energy by radiating, does not necessarily return to its initial entropy, certainly not until $\mathcal{U}$ equilibrates with the rest of the reservoir. But a (nonrotating uncharged) black hole whose mass has not changed overall, retains its original entropy because that depends only on mass. In addition, for the black hole mass and radius are related in a simple way; this allowed us to replace $T_{\mathrm{H}}$ in terms of $R$. By contrast, for a generic reservoir, size is not simply related to temperature.

Of course, the above derivation ignores the effect of Hawking radiation pressure. How important is this ? Could it blow $\mathcal{U}$ outwards ? If we approximate the radiance as black-body radiance of temperature $T_{\mathrm{H}}$ coming from a sphere of radius $2 M$, the energy flux at Schwarzschild coordinate $r$ from the hole is

$$
F(r)=\frac{\mathcal{N} \hbar}{61,440(\pi M r)^{2}},
$$

where $\mathcal{N}$ stands for the effective number of massless species radiated (photons contribute 1 to $\mathcal{N}$ and each neutrino species 7/16). This estimate is known to be off by 
a factor of only a few [24]. This energy (and momentum) flux results in a radiation pressure force $f_{\text {rad }}(r)=\pi R^{2} F(r)$ on $\mathcal{U}$. More precisely, species which reflect well off $\mathcal{U}$ are approximately twice as effective at exerting force as just stated, while those (neutrinos and gravitons) which go right through $\mathcal{U}$ contribute very little; the $\mathcal{N}$ must thus be reinterpreted accordingly. I have ignored relativistic corrections so that the result, as qualified, is correct mostly for $r \gg M$.

Writing the gravitational force on $\mathcal{U}$ in the Newtonian approximation, $f_{\text {grav }}(r)=$ $M E / r^{2}$, one sees that

$$
\frac{f_{\text {rad }}(r)}{f_{\text {grav }}(r)}=\frac{\mathcal{N}_{\text {eff }} \hbar R^{2}}{61,440 \pi^{2} M^{3} E}
$$

I have written $\mathcal{N}_{\text {eff }}$ here because, as mentioned, some species just pass through $\mathcal{U}$ without exerting force on it. In addition, only those species actually represented in the radiation flowing out during $\mathcal{U}$ 's infall have a chance to exert forces. Now an Hawking quantum bears an energy of order $T_{\mathrm{H}}$, so the number of quanta radiated together with energy $E$ is approximately $8 \pi M E / \hbar$. By our assumption that $\hbar / E<R$ and our stipulation that $M=\zeta R>R$, this is large compared to unity. Since a species can exert pressure only if it is represented by at least one quantum, one obviously has $\mathcal{N}_{\text {eff }}<8 \pi M E / \hbar$. Therefore,

$$
\frac{f_{\text {rad }}(r)}{f_{\text {grav }}(r)}<\frac{R^{2}}{7680 \pi M^{2}} \ll 1
$$

Radiation pressure is thus negligible, and $\mathcal{U}$ 's fall is very nearly on a geodesic, at least until $\mathcal{U}$ approaches to within a few Schwarzschild radii. It is intuitively clear that if $d \gg M$, the last (relativistic) stage cannot make any difference, and $\mathcal{U}$ must plunge to the horizon.

Whether $d$ is large enough must be checked. We have taken it such that the infall time equals the time $t$ for the hole to radiate energy $E$. Newtonially $d \approx 2\left(t^{2} M / \pi^{2}\right)^{1 / 3}$, while Eq. (10) gives the estimate $t \approx 5 \times 10^{4} E M^{2} \hbar^{-1} \mathcal{N}^{-1}$ with $\mathcal{N}$ now the full species number. From these equations and $M=\zeta R$ we get that $d \approx 1.2 \times 10^{3}(\zeta E R / \mathcal{N} \hbar)^{2 / 3} M$. Thus for $\mathcal{N}<10^{2}$ (conservative estimate of our world's massless particle content), we have $d>57 M$ for all systems $\mathcal{U}$ satisfying our assumption $R>\hbar / E$. Thus for all these we have established the entropy bound (9).

Our simple argument here leaves the factor $\nu \zeta$ somewhat fuzzy; but it is safe to say that $4 \nu \zeta<10^{2}$. Thus we recover bound (4) with some overshoot of the coefficient, not a large prize to pay for the simplicity of the derivation. When we come to strongly gravitating systems $(E \sim R)$, we cannot derive the bound (14) or even the weaker version (9) by the methods just expounded. Nevertheless, as mentioned, a black hole in $D=4$ spacetime saturates bound (雨) and complies with it for $D>4$. For strongly gravitating systems in asymptotically flat spacetime, the holographic bound and the entropy bound make very similar predictions for $D=4$, but for $D>4$ the holographic bound is the tighter of the two. Unless $D$ is very large, the entropy bound is the tighter bound for weakly gravitating systems, such as those we meet in everyday life. 


\subsection{Black holes as one--dimensional information conduits}

The holographic bound (1) is supposed to be telling us that a generic physical system in 4-D spacetime is fundamentally two-dimensional in space. It turns out that viewed as an information absorber or entropy emitter, a black hole in 4-D spacetime is fundamentally one-dimensional in space [30].

To show this one must define a one-dimensional information transmitting systema channel. In flat spacetime a channel is a complete set of one-way propagating modes of some field, with the modes enumerated by a single parameter. For example, all electromagnetic modes in free space with fixed wave vector direction and particular linear polarization constitute a channel; the modes are parametrized solely by frequency. One might implement such a channel with a straight infinitely long coaxial cable (which is well known to transmit all frequencies) capped at its entrance by the analog of a polaroid filter. Acoustic and neutrino channels can also be defined. A fundamental question is: what is the maximum rate, in quantum theory, at which information may be transmitted down such a channel for prescribed power $P$ ? The answer was found in the 1960's by several information theory pioneers (see my review with Schiffer 25]), but I want to reproduce here the much later but very simple derivation of Pendry [26], which is of very broad applicability.

Pendry thinks of a possible signal state as corresponding to a particular set of occupation numbers for the various propagating modes. He assumes the channel is uniform in the direction of propagation, which allows him to label the modes by momentum $p$. But he allows for dispersion, so that a quantum with momentum $p$ has some energy $\varepsilon(p)$. Then the propagation velocity of the quanta is the group velocity $v(p)=d \varepsilon(p) / d p$. Up to a factor ln 2 the information rate capacity is just the maximal one-way entropy current for given $P$, which obviously occurs for the thermal state, if one excludes the modes moving opposite the direction of interest.

Now the entropy $s(p)$ of any boson mode of momentum $p$ in a thermal state (temperature $T$ ) is 27]

$$
s(p)=\frac{\varepsilon(p) / T}{e^{\varepsilon(p) / T}-1}-\ln \left(1-e^{-\varepsilon(p) / T}\right),
$$

so the entropy current in one direction is

$$
\dot{S}=\int_{0}^{\infty} s(p) v(p) \frac{d p}{2 \pi \hbar}=\int_{0}^{\infty} s(p) \frac{d \varepsilon}{d p} \frac{d p}{2 \pi \hbar},
$$

where $d p / 2 \pi \hbar$ is the number of modes per unit length in the interval $d p$ which propagate in one direction. This factor, when multiplied by the group velocity, gives the one-way current of modes. Suppose $\varepsilon(p)$ is monotonic and extends over the range $[0, \infty)$; we may then cancel $d p$ and integrate over $\varepsilon$. Then after substitution of Eq. (13) and integration by parts we have

$$
\dot{S}=\frac{2}{T} \int_{0}^{\infty} \frac{\varepsilon}{e^{\varepsilon / T}-1} \frac{d \varepsilon}{2 \pi \hbar}=\frac{2}{T} \int_{0}^{\infty} \frac{\varepsilon(p)}{e^{\varepsilon(p) / T}-1} v(p) \frac{d p}{2 \pi \hbar} .
$$


The first factor in each integrand is the mean energy per mode, so that the integral represents the one-way power $P$ in the channel. Thus

$$
\dot{S}=2 P / T \text {. }
$$

The integral for $P$ in the first form of Eq. (15) can easily be done:

$$
P=\frac{\pi(T)^{2}}{12 \hbar}
$$

Eliminating $T$ between the last two expressions gives Pendry's limit

$$
\dot{S}=\left(\frac{\pi P}{3 \hbar}\right)^{1 / 2} \quad \text { or } \quad \dot{I}_{\max }=\left(\frac{\pi P}{3 \hbar}\right)^{1 / 2} \log _{2} e .
$$

For a fermion channel $P$ in Eq. (17) is a factor 2 smaller, and consequently $\dot{S}$ in Eq. (18) is reduced by a factor $\sqrt{ } 2$.

The function $\dot{S}(P)$ in Eq. (18) is the so called noiseless quantum channel capacity. Surprisingly it is independent, not only of the form of the mode velocity $v(p)$, but also of its scale. Thus the phonon channel capacity is as large as the photon channel capacity despite the difference in speeds. Why? Although phonons convey information at lower speed, the energy of a phonon is proportionately smaller than that of a photon in the equivalent mode. Thus when the capacities of channels harnessing various carriers are expressed in terms of power, they turns out to involve the same constants. Formula. (18) neatly characterizes what we mean by one-dimensional transmission of entropy or information. It refers to transmission by use of a single species of quantum and a specific polarization; different species and alternative polarizations engender separate channels. Although framed in a flat spacetime context, its lack of sensitivity to the dispersion relation of the transmitting milieu should make Pendry's limit relevant to curved spacetime also. This because electrodynamics in curved spacetime is equivalent to flat spacetime electrodynamics in a suitable dielectric and paramagnetic medium [28].

By contrast the power and entropy emission rate in a single boson polarization of a closed black body surface with temperature $T$ and area $A$ in flat $4-D$ spacetime is (half the Stefan-Boltzmann law)

$$
P=\frac{\pi^{2} T^{4} A}{120 \hbar^{3}} \quad \dot{S}=\frac{4}{3} \frac{P}{T}
$$

whereby

$$
\dot{S}=\frac{2}{3}\left(\frac{2 \pi^{2} A P^{3}}{15 \hbar^{3}}\right)^{1 / 4}
$$

[for fermions $P$ carries an extra factor $7 / 8$ and formula (20) an extra factor $(8 / 7)^{1 / 4}$ ]. Our manifestly 3-D transmission system deviates from the sleek formula (18) in the exponent of $P$ and in the appearance of the measure $A$ of the system. In emission 
from a closed curve of length $L$ in two-dimensional space, the factor $\left(L P^{2}\right)^{1 / 3}$ would replace $\left(A P^{3}\right)^{1 / 4}$. We may thus gather the dimensionality of the transmission system from the exponent of $P$ in the expression $\dot{S}(P)$ [it is $n /(n+1)$ for $D=n+1$ spacetime dimensions], as well as from the value of the coefficient of $P / T$ in expressions for $\dot{S}$ like (16) or (19) [it is $(n+1) / n$ ].

Radiation from a Schwarzschild black hole in 4-D spacetime is also given by Eqs. (19) (or their fermion version) with $A=4 \pi(2 M)^{2}$ and $T=T_{\mathrm{H}}$, except we must correct the expression for $P$ by a species dependent factor $\bar{\Gamma}$ of order unity [24], and replace the $4 / 3$ in the expression for $\dot{S}$ by the species dependent factor $\nu$ already mentioned in Sec. 1.3. Eliminating $M$ between the equations we obtain, in lieu of Eq. (20),

$$
\dot{S}=\left(\frac{\nu^{2} \bar{\Gamma} \pi P}{480 \hbar}\right)^{1 / 2}
$$

(For fermions there is an extra factor $7 / 8$ inside the radical). This looks completely different from the law (20) for the hot closed surface because, unlike for a hot body, a black hole's temperature is related to its mass.

However, (21) is of the same form as Pendry's limit (18) for one-channel transmission. From Page [24, 23\| we get $\bar{\Gamma}=1.6267$ and $\nu=1.5003$ for one photon polarization, so the numerical coefficient of (21) is 15.1 times that in (18). Repeating the above exercise for one species of neutrinos we again find formulae like (21) and (18), this time with $\bar{\Gamma}=18.045$ and $\nu=1.6391$; the numerical coefficient of (21) is 48.1 times that of the fermion version of (18).

Evidently in its entropy emission properties a black hole in 4-D spacetime is more like a 1-D channel than like a surface in 3-D space. Why is this ? A formal answer is that, because of the way $T_{\mathrm{H}}$ is related to the black hole's radius $2 M$, Hawking emission prefers to emerge in the lowest angular momentum mode possible. To exit with angular momentum $j \hbar$, a quantum must have energy (momentum) $\hbar \omega$ of order $j \hbar / 2 M$. But in the Hawking thermal distribution the dominant $\hbar \omega$ is of order $T_{\mathrm{H}}=\hbar(8 \pi M)^{-1}$. Thus the emerging $j$ 's tend to be small. For example, $97.9 \%$ of the photon energy emerges in the $j=1$ modes $(j=0$ is forbidden for photons), and $96.3 \%$ of the neutrino power is in the $j=\frac{1}{2}$ modes [24]. Thus the black hole emits as close to radially as possible. This means that, crudely speaking, it does so through just one channel.

\subsection{Information pulses in curved spacetime}

The discussion in Sec. 1.4 centered on steady state streams of information and energy. What if information is delivered as pulses ? Can one state a bound generalizing (18) ? Further, can one include effects of gravitation on information transfer rate ? To answer these questions let us extend the notion of channel to curved spacetime, at least to stationary curved spacetime. Again, a channel will be a complete set of one-way modes of some field that can be enumerated with a single parameter. Each channel is characterized by species of quanta, polarization (helicity), trajectory, etc. 
In Sec. 1.4 we characterized the signal in a particular channel by power. For a pulse it seems a better idea to use as signal parameters the signal's duration $\tau$ and its energy $E$. Since in curved spacetime a channel is not generally uniform, we choose to measure these parameters in a local Lorentz frame (we shall see presently that it does not matter which Lorentz frame). This precaution allows us to focus on sections of the channel and treat them as if we were working in flat spacetime.

How is $I_{\max }$ related to $E$ and $\tau$ ? Since information is dimensionless, $I_{\max }$ must be a function of dimensionless combinations of $E, \tau$, channel parameters and fundamental constants. We exclude channels which transmit massive quanta, e.g. electrons, because rest mass is energy in a form not useful for communication, so that the strictest limits on $I_{\max }$ should emerge for massless signal carriers. Hence Compton lengths do not enter into the argument. Also in order to maximize the information flux, we focus on broadband channels, and exclude any frequency cutoff. Finally, we exclude situations where the signal undergoes dispersion; this has the practical upshot that apart from light's speed $c$, only one other velocity - the signal velocity $c_{s}$ - can appear. We consider $c_{s} / c$ a property of the channel because it is common to all signals. If we temporarily exclude the gravitational constant, there is thus a single dimensionless combination of signal parameters, $\xi=E \tau / \hbar$, at our disposal. Thus

$$
I_{\max }=\Im(E \tau / \hbar)
$$

where $\Im(\xi)$ is some nonnegative valued function characteristic of the channel, the characteristic information function (CIF) 25].

Let us check formula (22) in flat spacetime in steady state (momentarily return to a long stream of information). Steady state means that the signal can be characterized as statistically stationary in a suitable frame. It should thus be possible to infer the peak information transfer rate by focusing on a finite section of the signal bearing information $I_{\max }$ and energy $E$. It should matter little how long a stretch in $\tau$ is used so long as it is not too short. This can only be true if $\dot{I}_{\max } \equiv E \tau^{-1}$ is fully determined by the power $P \equiv E \tau^{-1}$, and this is consistent with Eq. (22) only if $\Im(\xi) \propto \sqrt{ } \xi$ for only then does $\tau$ cancel out. We have thus recovered Pendry's limit (18), the correct answer for steady state; formula (22) checks out.

The dividing line between steady state information transfer and transfer by means of very long signals is not sharp. This suggests that long pulse signals must also obey a Pendry type formula, albeit approximately [29]. The law $\dot{I}_{\max } \propto(P / \hbar)^{1 / 2}$ is evidently inapplicable to brief information pulses. For such it may be replaced by a linear upper bound [31] which may even transcend some of the limitations we imposed to define $\Im(\xi)$. Consider the information $I$ to be encoded in some material structure $\mathcal{V}$ of radius $R$ and rest energy $E$ which maintains its integrity and dimensions as it flies from emitter to receiver. From Eq. (田) we have the strict inequality $I<2 \pi E R \hbar^{-1} \log _{2} e$. The rate at which the information is assimilated by the receiver is obviously restricted by the local time $\tau$ it takes for $\mathcal{V}$ to sweep by it. From special relativity $\tau>2 R \gamma^{-1}$ with $\gamma$ accounting for the Fitzgerald contraction of $\mathcal{V}$ in the frame of the receiver. 
Thus the peak information reception rate is $I / \tau<\pi E \hbar^{-1} \log _{2} e$, or

$$
\dot{I}_{\mathrm{rec}}<\pi E_{\mathrm{rec}} \hbar^{-1} \log _{2} e
$$

where $E_{\text {rec }} \equiv \gamma E$ is $\mathcal{V}$ 's energy as measured in the receiver's frame. This replaces the information version of Eq. (18) when it comes to pulses. With $\xi \equiv E_{\mathrm{rec}} \tau \hbar^{-1}$ we thus have the strict linear bound $\Im(\xi)<\left(\pi \log _{2} e\right) \xi$. There is a lot of evidence [32, 25] that this bound applies even when the signal has no rest frame. One should not be alarmed because the law $\Im(\xi) \propto \sqrt{ } \xi$ figuring in (18) exceeds the linear bound for small $\xi$; the law is meant for steady state, which makes sense only in the limit $\xi=E \tau / \hbar \rightarrow \infty$.

Let us check the local Lorentz invariance of (22). Consider a pair of local Lorentz frames, $\mathrm{A}$ and $\mathrm{B}$, encompassing a section of the channel, with $\mathrm{B}$ moving to the right with respect to $\mathrm{A}$ with speed $V<c_{s}$, and let $\gamma=\left(1-V^{2}\right)^{1 / 2}$. If there is a medium, $\mathrm{A}$ is taken as its rest frame, and $c_{s}$ is the signal's speed in this frame. Now let a right moving signal pulse's front (speed $c_{s}$ ) pass the origins of these frames at time $t_{\mathrm{A}}=0$ when they coincide. At a later time $t_{\mathrm{A}}=t_{1}$ the pulse's rear has reached A's origin; by then B's origin is at $x_{\mathrm{A}}=V t_{1}$. Sometime later, at $t_{\mathrm{A}}=t_{2}$, the pulse's rear reaches B's origin which is then at $x_{\mathrm{A}}=V t_{2}$. Calculating the pulse's length in frame $\mathrm{A}$ in two ways gives $\left(c_{s}-V\right) t_{2}=c_{s} t_{1}$, so that

$$
t_{1} / t_{2}=1-V / c_{s}
$$

The signal duration is $\tau_{\mathrm{A}}=t_{1}$ in $\mathrm{A}$; in $\mathrm{B}$, however, it is $\tau_{\mathrm{B}}=t_{2} \gamma^{-1}$ because $t_{2}$ is the time it takes in A for all the pulse to traverse B's origin; thus

$$
\tau_{\mathrm{A}}=\gamma \tau_{\mathrm{B}}\left(1-V / c_{s}\right)
$$

Now in A the energy and momentum of a (massless) quantum in the signal stand in the ratio $c_{s}$. Thus if interactions are negligible, the energy $E_{\mathrm{A}}$ and the corresponding momentum $P_{\mathrm{A}}$ of the full pulse stand also in the same ratio. Considering a Lorentz boost to frame $\mathrm{B}$ we discover that

$$
E_{\mathrm{B}}=\gamma\left(E_{\mathrm{A}}-V P_{\mathrm{A}}\right)=\gamma E_{\mathrm{A}}\left(1-V / c_{s}\right)
$$

Therefore, $E_{\mathrm{B}} \tau_{\mathrm{B}}=E_{\mathrm{A}} \tau_{\mathrm{A}}$. We can likewise prove this result for $1>V>c_{s}$. In view of this and the fact that information is a Lorentz scalar, the statement (22) is seen to be Lorentz invariant. This has immediate applications. For instance, A can be interpreted as the signal receiver's frame and B as the propagating medium's, or perhaps the transmitter's.

But how is the limit on information transmission rate related at two point along the channel ? In flat spacetime, and in the absence of dispersion, $E$ and $\tau$ are evidently conserved with propagation. So is the information, so that Eq. (22) is valid at every point along the channel. Once we are in stationary curved spacetime, $E$ and $\tau$ are subject to redshift and dilation effects, respectively. However, the effects act in 
opposite senses on $E$ and $\tau$, and since they depend on the same metric component, $E \tau$ is again conserved throughout the signal's flight. Therefore, Eq. (22) is meaningful throughout the channel. In fact one can use global values (as measured at infinity) of $E$ and $\tau$ in Eq. (22). We see that one and the same formula limits information transmission, propagation and reception rates.

We have so far excluded appearance of the gravitational constant in the formulae. This means we have been neglecting self-gravity of the signal, because this is measured by the parameter (momentarily restoring $G$ and $c$ ) $\varpi \equiv G E c^{-5} \tau^{-1}$, which is on the order of the ratio of the signal's self-potential energy to $E$, or that of the signal's gravitational radius to its duration. Should we include $\varpi$ as a new argument in Eq. (22) ? One reason for not doing so is that it would obviously spoil the local Lorentz invariance of Eq. (22) because $E / \tau$ is not a Lorentz scalar. We cannot allow this for signals propagating in vacuum in a flat background, for this would be tantamount to a violation of special relativity. However, it could be argued that the presence of $\varpi$ in $\Im$ is permissible for signals propagating in a medium $\left(c_{s} \neq c\right)$ because it locally defines a preferred Lorentz frame

In a curved background there are further arguments against inclusion of $\varpi$ in $\Im$. In vacuum we can use the requirement of local Lorentz invariance to bar $\varpi$ 's appearance, for a sufficiently brief signal should admit being encompassed in its entirety by local Lorentz frames. Further, $\varpi$ evidently decreases as the signal propagates outward in the gravitational potential. Thus, $\Im(E \tau / \hbar, \varpi)$ would decrease either outwardly (if $\Im$ increases with $\varpi$ ) or inwardly (if it decreases as $\varpi$ increases). If a signal's information saturates the bound $\Im(E \tau / \hbar, \varpi)$ at some point in the potential, then by conservation of information it will exceed the bound once it has propagated somewhat in the direction in which $\Im$ decreases. This leads to a contradiction. One could try to resolve the problem by defining $I_{\max }$ only in terms of the minimum value of $\varpi$ in the channel. But it seems strange that, at least for brief signals, one cannot state $I_{\text {nax }}$ in terms of local quantities.

It thus seems that for signals propagating in vacuum in curved spacetime, $\varpi$ cannot appear in $\Im$. It is unclear whether this conclusion extends to signal propagation in a medium, because in curved spacetime a medium is never homogeneous, which means, among other things, that $c_{s}$ varies. This in itself puts in doubt our argument for simplicity of the expression for $I_{\max }$.

\subsection{Dumping information into a black hole}

Suppose we have at our disposal a certain power $\mathcal{P}$ to accomplish the task of getting rid of a stream of possibly compromising information by dumping it into a black hole. We may pick the size of black hole which suits us best. By the complementary relation between entropy and information, formula (21) for entropy rate out of the black hole suggests a bound $\dot{I}_{\max } \sim(\mathcal{P} / \hbar)^{1 / 2}$ on the dumping rate. Here we verify this new bound.

First we argue that if the signal originates from afar, it is transmitted more or less 
through a single channel (per field species and polarization). Let us recall the rule for field mode counting. In one space dimension a length $L$ contains $(2 \pi)^{-1} L \Delta k$ modes in the wave vector interval $\Delta k$. In $3-\mathrm{D}$ space we would have $(2 \pi)^{-3} L_{x} L_{y} L_{z} \Delta k_{x} \Delta k_{y} \Delta k_{z}$ modes. From this we may conclude that if a flat 2 -surface of area $\mathcal{A}$ radiates into a narrow solid angle $\Delta \Omega$ about its normal, the number of modes out to a distance $L$ from it whose wave vector magnitudes lie between $k$ and $k+\Delta k$ is $(2 \pi)^{-3} \mathcal{A} L k^{2} \Delta \Omega \Delta k$. The factor $(2 \pi)^{-1} L \Delta k$ is obviously the number of modes emitted sequentially in each direction and distinguished by their values of $k$. One can thus think of $\mathcal{W}=$ $(2 \pi)^{-2} \mathcal{A} k^{2} \Delta \Omega$ as the number of active channels.

Now let a transmitter with effective area $\mathcal{A}$ send an information bearing signal towards a Schwarzschild black hole of mass $M$ surrounded by vacuum and situated at distance $d \gg 2 M$. Let $\mathcal{A}$ be oriented with its normal towards the black hole. Evidently, as viewed from the transmitter the black hole subtends solid angle $\Delta \Omega=$ $\pi(2 M)^{2} / d^{2}$, and we must have $\mathcal{A}<4 \pi d^{2}$. What should we take for $k$ in the formula for $\mathcal{W}$ ? Being interested in the highest information for given energy (other things being equal), we certainly want to use the smallest $k$ (smallest $\hbar \omega$ ) possible. But signals composed of too small $k$ 's will just be scattered by the black hole. The borderline is $k=2 \pi / \lambda \approx 2 \pi /(2 M)$. With this we find $\mathcal{W}<4 \pi^{2}$, which means that information transmission down a black hole is necessarily a few channels' affair (for each field species and polarization, of course) regardless of the scales $M$ and $d$ in the problem.

In light of this we employ formula (22). Further, since $E \tau$ is conserved in Schwarzschild (stationary) spacetime, and closely equals $\mathcal{E} t$, the values being measured at infinity, we have $I_{\max }=\Im(\mathcal{E} t / \hbar)$. This for a pulse of duration $t$ as seen from infinity. If we are dealing with a steady state stream of energy and information $(t \rightarrow \infty$ and $\mathcal{E} \rightarrow \infty$ with $\mathcal{P} \equiv \lim (\mathcal{E} / t)$ finite), we have by the logic of the paragraph following Eq. (22) that the maximum information disposal rate into the black hole is $\dot{I}_{\max } \sim(\mathcal{P} / \hbar)^{1 / 2}$. We have thus checked our guess. The precise proportionality factors for various fields may be worked out from Eq. (21). At any rate, we uncover a "cost" for information disposal into a black hole: the power required grows quadratically with the information dumping rate.

\section{Black Hole Spectroscopy}

\section{$2.1 \quad$ Introduction}

In classical general relativity we think of a black hole's parameters, typically charge $Q$, angular momentum $\mathbf{J}$ and mass $M$ (but others are possible: see Erick Weinberg's lectures in this collection) as continuous. In reality its angular momentum and charge should have discrete spectra. And what about $M$ ? Is it continuous as in the classical theory, or discrete as for an atom ? Granted that this is a fair question only for quantum gravity, it is still interesting to find out if something can be said about it in the fragmentary state of knowledge today. 
I have long argued [33] that certain features of classical black hole physics hint at a discrete mass spectrum of a very definite form. That would make black holes quite like atoms in one sense. Here I would like to summarize the various steps in the logic towards this conclusion, and describe an algebraic quantum formalism [34, 35, 36] designed to deal with this question irrespective of how the final quantum gravity may turn out. I shall use geometric units with $G=c=1 ; \sqrt{ } \hbar$ denotes the Planck mass.

\subsection{Adiabatic invariance and black hole quantization}

The present situation in quantum gravity's development, with rival theories (string theory, loop quantum gravity, canonical quantum gravity in several versions, ...) whose elements can still not be set in one to one correspondence is somewhat reminiscent of quantum physics before Schrodinger's equation and Heisenberg's matrix mechanics. People then were very much guided by the rules of the old quantum mechanics. And an important unifying principle of these was Ehrenfest's (Born's excellent book [37] is a convenient source): a quantity which classically is an adiabatic invariant has at the quantum level a discrete spectrum. The Bohr-Sommerfeld quantization rules, which focused on Jacobi actions $\oint p d q$ (all of which are adiabatic invariants), and which gave the correct hydrogen spectrum - fine structure includedexemplify the success of Ehrenfest's principle.

In the 1970's the work of Penrose and Floyd [38], Christodoulou [39] and Hawking 40] taught us that transformations of a classical black hole are generically irreversible because the surface area of the event horizon increases in most such changes. While the thermodynamic ramifications of this discovery have been of wide interest to the gravitation and particle communities for three decades, a less visible aspect of this purely mechanical behavior will be the center of attention here.

Christodoulou and Ruffini [39, 41] exhibited a set of black hole transformations which do not involve horizon area increase. The simplest example I can find deals with a point charge $\varepsilon$ which is deposited at rest, by whatever means, alongside the horizon of a Reissner-Nordström (RN) black hole with charge $Q$ of the same sign as $\varepsilon$. This means, purely on mechanical grounds, that the energy of the particle as measured at infinity, $E$, equals $\varepsilon \Phi$ with

$$
\Phi=\frac{Q}{M+\left(M^{2}-Q^{2}\right)^{1 / 2}}
$$

the electrostatic potential at the horizon. Now differentiation of the expression for horizon area of our Reissner-Nordström black hole, $A=4 \pi\left(M+\left(M^{2}-Q^{2}\right)^{1 / 2}\right)^{2}$, gives

$$
\begin{aligned}
\delta A & =(\delta M-\Phi \delta Q) \Theta^{-1} \\
\Theta & \equiv \frac{1}{2}\left(M^{2}-Q^{2}\right)^{1 / 2} A^{-1}
\end{aligned}
$$

By energy and charge conservation, when the particle is captured by the black hole, $\delta M=E$ while $\delta Q=\varepsilon$. But we selected $E=\varepsilon \Phi$, so that $\delta A=0$ upon capture of the 
particle. The process of charge assimilation is a slow one since the charge is gently placed on the horizon; and it results in a change of $M$ and $Q$ but with no consequent change in $A$. Hence $A$ is an adiabatic invariant here. Many other examples have been collected [42, 43, 44] involving the adiabatic addition of angular momentum to the black hole instead of charge, or various wave perturbations. In all these $A$ is unchanged.

The proposed adiabatic principle is a classical one. But if we take into account that by quantum theory the particle to be assimilated has an effective minimal radius (Compton length) which prevents us from placing its center exactly at the horizon without already loosing it, the same sort of calculation [11, 36] shows that the minimal horizon area increase is $(\delta A)_{\min }=\xi \hbar$, with $\xi$ a constant of $O(1)$. Adiabatic invariance is thus not literally true, but for super-Planckian black holes the area increase is relatively small:

$$
\left(\frac{\delta A}{A}\right)_{\min }=\frac{\xi \hbar}{A}<\frac{137 \xi e^{2}}{4 \pi Q^{2}}<\frac{137 \xi}{4 \pi}\left(\frac{\delta Q}{Q}\right)^{2} .
$$

Here we have used the fact that the elementary charge is of order $\sqrt{ } 137 \hbar^{1 / 2}$, that in all RN black holes $Q \leq(A / 4 \pi)^{1 / 2}$, and that the change in $Q$ cannot be less than $e$. Inequality (30) assures us that the fractional change in horizon area is small compared to that of black hole charge (pressumably $|\delta Q| \ll Q$ ), and generally also that of black hole mass because

$$
\frac{\delta M}{M}=\frac{\Phi \delta Q}{M}>\left(\frac{Q^{2}}{2 M^{2}}\right) \frac{\delta Q}{Q} .
$$

Thus in order for $(\delta A)_{\min } / A$ not to be small compared to $\delta M / M$, the black hole would have to be virtually neutral. In this sense we still have adiabatic invariance while allowing for quantum mechanics of matter. In fact, adiabatic invariance of $A$ seems to survive into the quantum gravity regime [45].

From the adiabatic invariance of the area of the horizon and Ehrenfest's principle, we should suspect that in quantum gravity the horizon area is replaced by an operator with a discrete spectrum. In the RN example the classical formula for $A$ gives $M=(A / 16 \pi)^{1 / 2}\left[1+4 \pi Q^{2} / A\right]$. If this were true for operators, the discrete spectra of $Q$ and $A$ would imply that $M$ too has a discrete spectrum [35]. Of course, quantum corrections to this last formula might come about [46] without eliminating the discreteness of $M$. And if the area eigenvalues are themselves degenerate, quantum corrections might split this degeneracy [35, 36]. However, it seems most constructive, in view of the absence of a consensus quantum gravity theory, to consider the pristine situation before all these corrections are effective.

\subsection{Dynamical variables and creation operators}

In quantum theory $\mathbf{J}$ and $Q$ are represented by hermitian operators $\hat{\mathbf{J}}$ and $\hat{Q}$. We

assume $[\hat{\mathbf{J}}, \hat{Q}]=0$ so that the black hole can simultaneously have sharp charge, which we assume to be an integer multiple of $e$, and angular momentum. In order to get 
usual spectrum $\mathbf{J}^{2}=j(j+1) \hbar^{2} ; j=0, \frac{1}{2} \hbar, \hbar \cdots$ with $J_{z} \equiv m \hbar=\{-j,-j+1, \cdots, j\} \hbar$, we have to assume that $\hat{\mathbf{J}} \times \hat{\mathbf{J}}=\imath \hbar \hat{\mathbf{J}}$. The argument at the end of Sec. 2.2 predisposes us to expect that there is some observable representing horizon area, $A$, which has a simple spectrum, $\left\{a_{1}, a_{2}, \cdots a_{n} \cdots\right\}$ with $a_{n+1}>a_{n}$. Now the horizon area of a black hole is invariant under rotations of its spin; since $\hat{\mathbf{J}}$ is the generator of such rotations in quantum theory, one expects that $[\hat{A}, \hat{\mathbf{J}}]=0$. Similarly, horizon area is invariant under gauge transformations; in quantum theory their generator is, as usual, the charge $\hat{Q}$. Hence we expect that $[\hat{A}, \hat{Q}]=0$.

It follows that we can conceive of a basis of one-black hole states of the form $\{|n j m q s\rangle\}$ where $q$ is an integer eigenvalue of $\hat{Q} / e$, and $s$ distinguishes between the different black hole states with like $a_{n}, j, m$ and $q$ (this degeneracy is really a must; see Sec. 2.6). But the above algebra of observables is too simple, and cannot by itself tell us very much new. It has to be extended. Gour 46] has shown how to introduce a "secret" operator corresponding to the quantum number $s$; his prescription, however, presupposes a uniformly spaced spectrum for $\hat{A}$, which is an idea to be tested here. We, therefore, avoid including something like this in the algebra.

In field theory of particles we would at this point introduce fields and analyze them into creation and anhilation operators; but "field" is an inappropriate concept for black holes which are not even approximately pointlike objects. Nobody stops us, however, from defining the black hole vacuum $\mid$ vac $\rangle$ (spacetime with no black holes or particles of any sort), and creation operators for black holes $\hat{R}_{n j m q s}$ with the property that $|n j m q s\rangle=\hat{R}_{n j m q s}|\mathrm{vac}\rangle$. This is essentially a tautology, not a physical assumption, because we introduce as many operators as there are states. In contrast to field theory, we do not assume that $\hat{R}_{n j m q s}{ }^{N}$ creates a state with $N$ black holes.

By commuting $\hat{A}, \hat{\mathbf{J}}$ and $\hat{Q}$ with the $\hat{R}_{n j m q s}$ and iterating we can make more operators. If this process continues indefinitely, no information can be obtained from the algebra unless additional assumptions are made. So let us suppose that the mentioned operators, together with the unit operator $\hat{I}$, form a closed, linear, infinite dimensional algebra. This assumption has two new features: the closure at some low level of commutation, essentially a plea for simplicity, and the linear character of the algebra when formulated in terms of $\hat{A}$. As we shall see in Sec. 2.5, this last implies the additivity of horizon area, which is a reasonable property. By contrast, additivity of mass for two black holes is not reasonable (nonlinearity of gravity), and this is really the reason why one cannot assume linearity of the algebra of $\hat{M}, \hat{Q}, \hat{\mathbf{J}}$ and $\hat{R}_{n j m q s}$. In this sense $\hat{A}$ is special among all functions of the other black hole observables.

By definition $\hat{R}_{n j m q s} \mid$ vac $\rangle$ is an eigenstate of $\hat{Q}$ with eigenvalue qe, so

$$
\exp (\imath \chi \hat{Q}) \hat{R}_{n j m q s}|\operatorname{vac}\rangle=\exp (\imath \chi q e) \hat{R}_{n j m q s}|\operatorname{vac}\rangle
$$

for real $\chi$. Thus, as already mentioned, $\hat{Q}$ is the generator of (global) quantum gauge transformations, and this is equivalent to requiring

$$
\exp (\imath \chi \hat{Q}) \hat{R}_{n j m q s} \exp (-\imath \chi \hat{Q})=\exp (\imath \chi q e) \hat{R}_{n j m q s}
$$


(check by operating with this on $|\mathrm{vac}\rangle$, recalling that $\hat{Q}|\mathrm{vac}\rangle=0$, and recovering Eq. (32)). Now expansion of Eq. (33) to $O(\chi)$ gives one of our essential commutators,

$$
\left[\hat{Q}, \hat{R}_{n j m q s}\right]=q e \hat{R}_{n j m q s} \text {. }
$$

Now obviously

$$
\exp \left(\imath \chi \hat{J}_{z} / \hbar\right) \hat{R}_{n j m q s}|\operatorname{vac}\rangle=\exp (\imath \chi m) \hat{R}_{n j m q s}|\operatorname{vac}\rangle,
$$

Repeating the previous sort of argument we get

$$
\left[\hat{J}_{z}, \hat{R}_{n j m q s}\right]=m \hbar \hat{R}_{n j m q s}
$$

There is a more significant way to get Eq. (36). Since $\hat{R}_{n j m q s} \mid$ vac $\rangle$ is defined as a state with spin quantum numbers $j$ and $m$, the collection of such states with fixed $j$ and all allowed $m$ must transform among themselves under rotations of the black hole like the spherical harmonics $Y_{j \mu}$ (or the corresponding spinorial harmonic when $j$ is half-integer). But $\mid$ vac $\rangle$ must obviously be invariant under all rotations, so $\hat{R}_{n j m q s}$ may be taken to behave like an irreducible spherical tensor operator of rank $j$ with the usual $2 j+1$ components labeled by $m$ 47. This gives Eq. (36) immediately. In addition, defining the usual raising and lowering operators, $\hat{J}_{ \pm} \equiv J_{x} \pm \imath J_{y}$, we infer

$$
\left[\hat{J}_{ \pm}, \hat{R}_{n j m q s}\right]=\sqrt{j(j+1)-m(m \pm 1)} \hbar \hat{R}_{n j, m \pm 1, q s} .
$$

We can use this and the identity $47 \hat{\mathbf{J}}^{2}=(1 / 2)\left(\hat{J}_{+} \hat{J}_{-}+\hat{J}_{-} \hat{J}_{+}\right)+\hat{J}_{z}^{2}$ to work out $\left[\hat{\mathbf{J}}^{2}, \hat{R}_{\kappa s}\right]$. It has a rather complicated form; its first term is $j(j+1) \hbar^{2} \hat{R}_{n j m q s}$ which is followed by two terms having on their right hand sides $\hat{J}_{+}$and $\hat{J}_{-}$, respectively. Thus operating with $\left[\hat{\mathbf{J}}^{2}, \hat{R}_{n j m q s}\right]$ on $\mid$ vac $\rangle$ and taking into account that $\hat{\mathbf{J}} \mid$ vac $\rangle=0$ we get

$$
\hat{\mathbf{J}}^{2} \hat{R}_{n j m q s}|\operatorname{vac}\rangle=j(j+1) \hbar^{2} \hat{R}_{n j m q s}|\mathrm{vac}\rangle
$$

This corresponds to the definition of $\hat{R}_{n j m q s}$ as creation operator of a black hole with angular momentum quantum numbers $j$ and $m$.

\subsection{Including $\hat{A}$ in the algebra}

So far all we know about $\hat{A}$ is that it commutes with $\hat{\mathbf{J}}$ and $\hat{Q}$. We can extend the algebra to it by using the Jacobi identity

$$
[\hat{W},[\hat{V}, \hat{U}]]+[\hat{V},[\hat{U}, \hat{W}]]+[\hat{U},[\hat{W}, \hat{V}]]=0
$$

Replacing $\hat{W} \rightarrow \hat{A}, \hat{U} \rightarrow \hat{R}_{n j m q s}$, and $\hat{V}$ in turn by $\hat{J}_{z}, \hat{J}_{ \pm}$and $\hat{Q}$, and using Eqs. (34), (36) and (37) as well as the mutual commutativity of all observables, we obtain

$$
\begin{aligned}
& {\left[\hat{Q},\left[\hat{A}, \hat{R}_{n j m q s}\right]\right]=q e\left[\hat{A}, \hat{R}_{n j m q s}\right] .} \\
& {\left[\hat{J}_{z},\left[\hat{A}, \hat{R}_{n j m q s}\right]=m \hbar\left[\hat{A}, \hat{R}_{n j m q s}\right]\right.} \\
& {\left[\hat{J}_{ \pm},\left[\hat{A}, \hat{R}_{n j m q s}\right]\right]=\sqrt{j(j+1)-m(m \pm 1)} \hbar\left[\hat{A}, \hat{R}_{n j, m \pm, q s}\right] .}
\end{aligned}
$$


The fact that these commutators mimic those in Eqs. (34), (36) and (37) properly reflects the rotational and gauge invariant status of $\hat{A}$ which forces $\left[\hat{A}, \hat{R}_{n j m q s}\right]$ to transform exactly like $\hat{R}_{n j m q s}$. Note that all the previous commutation relations are invariant under the redefinition $\hat{A} \rightarrow \hat{A}+$ const. We single out the $\hat{A}$ of physical interest by the requirement that $\hat{A}|\mathrm{vac}\rangle=0$.

According to our closure assumption, $\left[\hat{A}, \hat{R}_{n j m q s}\right]$ has to be a linear combination of some of the operators $\hat{I}, \hat{R}_{n^{\prime} j^{\prime} m^{\prime} q^{\prime} s^{\prime}}, \hat{A}, \hat{Q}, \hat{J}_{z}$ and $\hat{J}_{ \pm}$. From Eq. (33) and its analog for $\hat{J}_{z}$ and rotations, it is clear that $\hat{I}, \hat{Q}$ and $\hat{A}$, all of them rotational scalars and gauge invariants, can only show up in the linear combination in the cases $\{n q j m s\}=$ $\{n 000 s\}$. Further, the triplet $\left\{\hat{J}_{-1}, \hat{J}_{0}, \hat{J}_{+1}\right\} \equiv\left\{\hat{J}_{-}, \hat{J}_{z}, \hat{J}_{+}\right\}$is gauge invariant and a spherical irreducible tensor of rank one [47], so $\hat{J}_{\nu}$ with $\nu=0, \pm 1$ can only show up in the linear combination in the cases $\{n q j m s\}=\{n 01 \nu s\}$. Furthermore, by rotational invariance all three $\hat{J}_{\nu}$ must occur with like coefficient. Finally, $\hat{R}_{n^{\prime} j^{\prime} m^{\prime} q^{\prime} s^{\prime}}$ can only show up if its subscripts $q^{\prime}, j^{\prime}$ and $m^{\prime}$ match those of $\left[\hat{A}, \hat{R}_{n j m q s}\right]$ (we cannot have another $j$ appearing since Eq. (42) makes it clear that $\left[\hat{A}, \hat{R}_{n j m q s}\right]$ contains a single $j)$. In equations

$$
\left[\hat{A}, \hat{R}_{n j m q s}\right]=\sum_{n^{\prime} s^{\prime}} h_{n s}^{n^{\prime} s^{\prime}} \hat{R}_{n^{\prime} j m q s^{\prime}}+\delta_{q}{ }^{0}\left[\delta_{j}^{0}\left(C_{n s} \hat{I}+D_{n s} \hat{Q}+E_{n s} \hat{A}\right)+\delta_{j}{ }^{1} F_{n s} \hat{J}_{m}\right]
$$

where $C_{n s}, D_{n s}, E_{n s}$ and $F_{n s}$ are c-numbers and $h_{n s}^{n^{\prime} s^{\prime}}$ is a c-number matrix.

Let us now operate with Eq. (43) on the vacuum; $\hat{Q}, \hat{\mathbf{J}}$ and $\hat{A}$ all anhilate it, so we are left with

$$
a_{n} \hat{R}_{n j m q s}|\mathrm{vac}\rangle=\sum_{n^{\prime} s^{\prime}} h_{n s}^{n^{\prime} s^{\prime}} \hat{R}_{n^{\prime} j m q s^{\prime}}|\mathrm{vac}\rangle+\delta_{q}^{0} \delta_{j}^{0} C_{n s}|\mathrm{vac}\rangle
$$

However, it is clear that the states $\{|n q j m s\rangle\}$ are orthogonal to one another (automatically in the space spanned by $n, q, j, m$ and by Schmidt orthogonalization with respect to the $s$ quantum number), and all of them to the vacuum. The previous equation will contradict this unless we demand $C_{n s}=0$ and $h_{n s}^{n^{\prime} s^{\prime}}=a_{n} \delta_{n}{ }^{n^{\prime}} \delta_{s}{ }^{s^{\prime}}$. In that case

$$
\left[\hat{A}, \hat{R}_{n j m q s}\right]=a_{n} \hat{R}_{n j m q s}+\delta_{q}{ }^{0}\left[\delta_{j}{ }^{0}\left(D_{n s} \hat{Q}+E_{n s} \hat{A}\right)+\delta_{j}{ }^{1} F_{n s} \hat{J}_{m}\right] .
$$

At this stage we exploit the freedom left in $\hat{R}_{n j m q s}$ to define a new set of creation operators

$$
\hat{\mathcal{R}}_{n j m q s} \equiv \hat{R}_{n j m q s}+\left(a_{n}\right)^{-1} \delta_{q}{ }^{0}\left[\delta_{j}{ }^{0}\left(D_{n s} \hat{Q}+E_{n s} \hat{A}\right)+\delta_{j}{ }^{1} F_{n s} \hat{J}_{m}\right]
$$

After the redefinition the algebra of $\left\{\hat{I}, \hat{A}, \hat{Q}, \mathbf{J}, \hat{\mathcal{R}}_{n j m q s}\right\}$ is still closed, and the $\hat{\mathcal{R}}_{n j m q s}$ create exactly the same states as the $\hat{R}_{n j m q s}$. The redefinition transforms Eqs. (45) and (34) into

$$
\begin{aligned}
& {\left[\hat{A}, \hat{\mathcal{R}}_{n j m q s}\right]=a_{n} \hat{\mathcal{R}}_{n j m q s}} \\
& {\left[\hat{Q}, \hat{\mathcal{R}}_{n j m q s}\right]=q \hat{\mathcal{R}}_{n j m q s},}
\end{aligned}
$$

but changes the forms of (36) and (37) slightly [48. 


\subsection{The area spectrum}

What is the state $\hat{\mathcal{R}}_{n j m q s}\left|n^{\prime} q^{\prime} j^{\prime} m^{\prime} s^{\prime}\right\rangle=\hat{\mathcal{R}}_{n j m q s} \hat{\mathcal{R}}_{n^{\prime} q^{\prime} j^{\prime} m^{\prime} s^{\prime}} \mid$ vac $\rangle$ ? In field theory we would unhesitatingly identify it as a two-black hole state. Here it is different. To explain why, it is useful to denote the quantum numbers $\{n j m q s\}$ collectively by a Greek index, $\kappa, \lambda$ or $\mu$, as the case may be. Likewise, we denote the operators $\hat{Q}$ and $\hat{A}$ by the common symbol $\hat{X}$, and the latter's eigenvalues by $\xi_{\lambda}$. From Eqs. (47)-(48) and the Jacobi identity (39) we discover that

$$
\left[\hat{X},\left[\hat{\mathcal{R}}_{\kappa}, \hat{\mathcal{R}}_{\lambda}\right]\right]=\left(\xi_{\kappa}+\xi_{\lambda}\right)\left[\hat{\mathcal{R}}_{\kappa}, \hat{\mathcal{R}}_{\lambda}\right]
$$

Now by the closure condition

$$
\left[\hat{\mathcal{R}}_{\kappa}, \hat{\mathcal{R}}_{\lambda}\right]=\sum_{\mu} \varepsilon_{\kappa \lambda}^{\mu} \hat{R}_{\mu}+\cdots
$$

where the ellipsis signifies some linear combination of $\hat{A}, \hat{J}_{m}$ and $\hat{Q}$, and $\varepsilon_{\kappa \lambda}^{\mu}$ are c-numbers (structure constants). Substituting in Eq. (49) gives

$$
\sum_{\mu} \varepsilon_{\kappa \lambda}^{\mu}\left[\hat{X}, \mathcal{R}_{\mu}\right]=\left(\xi_{\kappa}+\xi_{\lambda}\right)\left(\sum_{\mu} \varepsilon_{\kappa \lambda}^{\mu} \hat{\mathcal{R}}_{\mu}+\ldots\right),
$$

which in view of Eqs. (47)-(48) excludes the $\cdots$ terms, and also tells us that whenever $\varepsilon_{\kappa \lambda}^{\mu} \neq 0$,

$$
\xi_{\mu} \equiv \xi_{\kappa}+\xi_{\lambda}
$$

for both types of $\hat{X}$. We shall assume that for given $\kappa$ and $\lambda$ at least one of the $\varepsilon_{\kappa \lambda}^{\mu} \neq 0$ does not vanish (see below for the interpretation).

Operating on $\mid$ vac $\rangle$ with Eq. (50) one gets

$$
\left[\hat{\mathcal{R}}_{\kappa}, \hat{\mathcal{R}}_{\lambda}\right]|\mathrm{vac}\rangle=|\bullet\rangle
$$

where $|\bullet\rangle$ stands for a one-black hole state, a superposition of states $\{n j m q s\}$ which by virtue of Eq. (49) all have a common area eigenvalue $a_{\kappa}+a_{\lambda}$ and a common charge $q_{\kappa}+q_{\lambda}$. This $|\bullet\rangle$ is obviously a physical state (it complies with the charge superselection rule); it may involve superpositions of different $j$ and $m$.

We conclude that

$$
\left.|\Psi\rangle \equiv \hat{\mathcal{R}}_{\kappa} \hat{\mathcal{R}}_{\lambda} \mid \text { vac }\right\rangle=\frac{1}{2}|\bullet \bullet\rangle+\frac{1}{2}|\bullet\rangle .
$$

Here $|\bullet \bullet\rangle=\left(\mathcal{R}_{\kappa} \mathcal{R}_{\lambda}+\mathcal{R}_{\lambda} \mathcal{R}_{\kappa}\right) \mid$ vac $\rangle$ is obviously a two-black hole state symmetric under exchange of the $\kappa$ and $\lambda$ sets of quantum numbers. Thus trying to add an extra black hole to the state $\{n j m q s\}$ actually creates a linear combination of a one- and a two-black hole states. This is reasonable since classically black holes can merge, so there is some amplitude for the new black hole to fuse with the original one. This is the ultimate justification for our assumption that at least one of the $\varepsilon_{\kappa \lambda}^{\mu}$ is nonzero. 
Now using Eqs. (47)-(48) twice we find that

$$
\hat{X}|\Psi\rangle=\hat{\mathcal{R}}_{\kappa}\left(\hat{X}+\xi_{\kappa}\right) \hat{\mathcal{R}}_{\lambda}|\operatorname{vac}\rangle=\left(\xi_{\kappa}+\xi_{\lambda}\right)|\Psi\rangle
$$

Thus the states $|\Psi\rangle$ and $|\bullet \bullet\rangle$ both have sharp area $a_{\kappa}+a_{\lambda}$ and sharp charge $q_{\kappa}+q_{\lambda}$, just like $|\bullet\rangle$ : area, like charge, is an additive quantity for two black holes. This additivity jibes with our geometric notion that areas of separate objects are additive, and serves as further justification for the assumptions leading to our algebra. And because $|\Psi\rangle$ involves $|\bullet\rangle$, we find, in addition, that the sum of two eigenvalues of $\hat{Q}$ or $\hat{A}$ for a single black hole is also a possible eigenvalue of $\hat{Q}$ or $\hat{A}$, respectively, of a single black hole.

It turns out that differences of eigenvalues of the $\hat{X}$ are also eigenvalues for one black hole. Consider the hermitian conjugates of Eqs. (47)-(48),

$$
\left[\hat{X}, \hat{\mathcal{R}}_{\kappa}^{\dagger}\right]=-\xi_{\kappa} \hat{\mathcal{R}}_{\kappa}^{\dagger}
$$

What is the meaning of $|\chi\rangle \equiv \hat{\mathcal{R}}_{\kappa}^{\dagger}|\mathrm{vac}\rangle$ ? Operating with Eq. (56) with $\hat{X}=\hat{A}$ on $\mid$ vac $\rangle$ and taking the scalar product with $\langle\chi|$ shows that $\hat{A}$ would have a negative average in the state $|\chi\rangle$, unless this last state vanishes. Thus, since $\hat{A}$ is a positive definite operator, $\hat{\mathcal{R}}_{\kappa}^{\dagger}$ must anhilate the vacuum. It seems very plausible then that $|\Xi\rangle \equiv \hat{\mathcal{R}}_{\kappa}^{\dagger} \hat{\mathcal{R}}_{\lambda}|\mathrm{vac}\rangle$ can only be a one-black hole state, pressumably distinct from $\hat{\mathcal{R}}_{\lambda}|\mathrm{vac}\rangle$. Applying Eqs. (47),(48) and (56) gives

$$
\hat{X}|\Xi\rangle=\left(\hat{\mathcal{R}}_{\kappa}^{\dagger} \hat{X}-\xi_{\kappa} \hat{\mathcal{R}}_{\kappa}^{\dagger}\right) \hat{\mathcal{R}}_{\lambda}|\operatorname{vac}\rangle=\left(\xi_{\lambda}-\xi_{\kappa}\right)|\Xi\rangle .
$$

This verifies our claim, with the obvious caveat that if $a_{\lambda}<a_{\kappa}, \hat{\mathcal{R}}_{\kappa}^{\dagger}$, must anhilate the state $\hat{\mathcal{R}}_{\lambda}|\mathrm{vac}\rangle$ because negative black hole areas are unacceptable (the same must happen if $a_{\lambda}=a_{\kappa}$ with $q_{\lambda} \neq q_{\kappa}$ because a zero area state is necessarily the vacuum, which bears no charge. Hence the positive difference of two eigenvalues of $\hat{A}$ for a single black hole is also a possible eigenvalue of $\hat{A}$ of a single black hole; the difference of two $\hat{Q}$ eigenvalues is a possible $\hat{Q}$ eigenvalue for one black hole.

We take it from Sec. 2.2 that the spectrum of $\hat{A}$ is discrete. Now the only discrete set of positive real numbers that is unchanged under addition or absolute value substraction of two members is the set of all the natural numbers multiplied by some common factor. We conclude that the one-black hole area spectrum is just $\left\{n a_{1} \mid n=1,2, \cdots\right\}$, where $a_{1}$ is some positive scale of area (here we make no attempt to determine which eigenvalues correspond to which charges and spin; see [48]). It is also clear that the two rules (in italics) lead to a one-black hole charge spectrum composed of all integers multiplied by a common factor, e. We have thus formally obtained from the algebra the kind of area spectra predicted long ago [33], and summarized in Sec. 2.2. For nonrotating neutral black holes, the corresponding mass eigenvalues are proportional to $\{\sqrt{ } n \mid n=1,2, \ldots\}$.

Based on a variety of interpretations of the nonrotating neutral black hole in canonical quantum gravity, Schiffer (whose paper's title is used as title of the present 
lecture), Peleg, Kastrup, Louko and Mäkelä, Barvinsky and Kunstatter, Berezin, Vaz and Witten 49] have all obtained a mass spectrum of the mentioned form, but with no consensus as to the exact numerical coefficient. Some such calculations give a not uniformly spaced spectrum [50. Regarding RN black holes, Mäkelä and Repo 51 find the sum of areas of outer and inner horizons to scale like an integer, and a recent paper of theirs and coworkers extends this rule to charged rotating black holes [52]. Vaz and Witten [53 find a law of this form rather for the difference of these areas. And Barvinsky, Das and Kunstatter 45] find an external horizon area with a spectrum precisely equally spaced, but with its zero point shifted by a charge dependent quantity. Overall, the predictions are thus similar to those following from the algebra.

\subsection{Degeneracy of area eigenvalues}

According to Mukhanov [54] degeneracy plays a central role in any discussion of area levels. What can we say about it from our approach? By rotational invariance neither area eigenvalues nor degeneracy factors can depend on the quantum number $m$. Let us assume that the spectrum $a_{n}=n a_{1}$ for $n=1,2, \cdots$ is common to every combination of quantum numbers $j$ and $q$ (there is one alternative to this [48]). The degeneracy factor will be of the form $g_{n}=g_{n}(j, q)$. Now for fixed $\left\{n_{\kappa}, j_{\kappa}, m_{\kappa}, q_{\kappa}\right\}$ with not all of $j_{\kappa}, m_{\kappa}$ and $q_{\kappa}$ vanishing, there are $g_{n_{\kappa}}\left(j_{\kappa}, q_{\kappa}\right)$ independent one-black hole states $\hat{\mathcal{R}}_{\kappa}|\mathrm{vac}\rangle$ distinguished by the values of $s$. Analogously, the set $\left\{n_{\lambda}=1, j_{\lambda}=0, m_{\lambda}=\right.$ $\left.0, q_{\lambda}=0\right\}$ specifies $g_{1}(0,0)$ independent states $\hat{R}_{\lambda} \mid$ vac $\rangle$, all different from the previous ones because not all quantum numbers agree. One can thus form $g_{1}(0,0) \cdot g_{n_{\kappa}}\left(j_{\kappa}, q_{\kappa}\right)$

one-black hole states, $\left[\hat{R}_{\kappa}, \hat{R}_{\lambda}\right] \mid$ vac $\rangle$, with area eigenvalues $\left(n_{\kappa}+1\right) a_{1}$, and charge and spin just like the states $\hat{R}_{\kappa} \mid$ vac $\rangle$. Let us assume these new states are independent. Then their number cannot exceed the total number of states with area $\left(n_{\kappa}+1\right) a_{1}$ and quantum numbers $j_{\kappa}$ and $q_{\kappa}: g_{n_{\kappa}+1}\left(j_{\kappa}, q_{\kappa}\right) \geq g_{1}(0,0) \cdot g_{n_{\kappa}}\left(j_{\kappa}, q_{\kappa}\right)$. Iterating this inequality starting from $n_{\kappa}=1$ gives (we drop $\kappa$ )

$$
g_{n}(j, q) \geq g_{1}(j, q) \cdot g_{1}(0,0)^{n-1} .
$$

If $g_{1}(0,0) \neq 1$, Eq. (58) tells us that the degeneracy rises at least exponentially with area. Since the area spectrum is rather sparse, black hole entropy must receive its principal contribution from the logarithm of the degeneracy of area levels, so what we have just found is that black hole entropy must grow at least as fast as the horizon area. Thus we have a pleasant microscopic explanation of the black hole entropy-area relation. Further, as first emphasized by Mukhanov [54, one can calibrate the area spectrum by use of the degeneracy $\leftrightarrow$ entropy correspondence relation. For $g_{1}(0,0)=2$ this gives $a_{1}=4 \hbar \ln 2$ with the corresponding mass spectrum $\left\{(\hbar \ln 2 / 4 \pi)^{1 / 2} \sqrt{ } n \mid n=1,2, \cdots\right\}$ [55]. 


\section{Acknowledgments}

This research is supported by grant No. 129/00-1 of the Israel Science Foundation.

\section{References}

[1] Hooft, G. 't (1993) in Aly, A., Ellis, J. and Randjbar-Daemi, S. (eds.), Salamfestschrifft, World Scientific, Singapore, gr-qc/9310026.

[2] Susskind, L. (1995) J. Math. Phys., 36, 6377.

[3] Bekenstein, J.D. (1974) Lett. Nuovo Cimento, 4, 737 and Phys. Rev. D, 9, 3292.

[4] Wald, R.M. (1999) preprint hep-th/9912119.

[5] Bekenstein, J.D. (2000) Phys. LettersB, 481, 339; (2001) in Ruffini, R. (ed.), Proceedings of the Ninth Marcel Grossmann Meeting on General Relativity, World Scientific, Singapore.

[6] Bousso, R. (1999) J. High Energy Phys., 9906, 028.

[7] Peres, A. (1993) Quantum Theory: Concepts and Methods, Kluwer, Dordrecht.

[8] Shannon, C. and Weaver, W. (1949) The Mathematical Theory of Communication, Univ. of Illinois Press, Urbana.

[9] Katz, A. (1967) Principles of Statistical Mechanics, Freeman, San Francisco.

[10] Bekenstein, J.D. (1981) Phys. Rev. D, 23, 287.

[11] Bekenstein, J.D. (1973) Phys. Rev. D, 7, 2333.

[12] Unruh, W.G. and Wald, R.M. (1982) Phys. Rev. D, 25, 942 and (1983) Phys. Rev. D, 27, 2271; Pelath, M.A. and Wald, R.M. (1999) Phys. Rev. D, 60, 104009.

[13] Bekenstein, J.D. (1982) Phys. Rev. D, 26, 950; (1983) Phys. Rev. D, 27, 2262.

[14] Bekenstein, J.D. (1994) Phys. Rev. D, 49, 1912; (1999) Phys. Rev. D, 60, 124010.

[15] Zaslavskii, O. (1991) Phys. Lett. A, 160, 339.

[16] Hod, S. (1999) Phys. Rev. D, 60, 104031; (2000) Phys. Rev. D, 61, 024018 and 61, 024023.

[17] Mayo, A.E. (1999) Phys. Rev. D, 60, 104044; Bekenstein, J.D. and Mayo, A.E. (2000) Phys. Rev. D, 61, 024022. 
[18] Bekenstein, J.D. (1996) in Jantzen, R.T. and Keiser, G.M. (eds.), Proceedings of the VII Marcel Grossmann Meeting on General Relativity, World Scientific, Singapore, gr-qc/9409015.

[19] Bousso, R. (2001) J. High Energy Phys., 0104, 035.

[20] Verlinde, E. (2000) preprint hep-th/0008140.

[21] Cardy, J. L. (1986) Nucl. Phys. B, 270, 317.

[22] Bekenstein, J.D. (1989) Int. J. Theor. Phys., 28, 967; Schiffer, M. (1991) Int. J. Theor. Phys., 30, 419; Fischler, W. and Susskind, L. (1998) preprint hepth/9806039; Bousso, R. (1999) J. High Energy Phys., 9907, 004; Easther R. and Lowe, D. (1999) Phys. Rev. Letters, 82, 4967; Veneziano, G. (1999) Phys. Lett. B, 454, 22; Kaloper, N. and Linde, A. (1999) Phys. Rev. D, 60, 103509; Bak D. and Rey, S.-J. (2000) Class. Quant. Grav., L83, 17; Brustein, R. and Veneziano, G. (2000) Phys. Rev. Letters, 84, 5695.

[23] Page, D.N. (1983) Phys. Rev. Letters, 50, 1013.

[24] Page, D.N. (1976) Phys. Rev. D, 13, 198.

[25] Bekenstein, J.D. and Schiffer, M. (1990) Int. J. Mod. Physics C, 1, 355.

[26] Pendry, J.B. (1983) J. Phys. A, 16, 2161.

[27] Landau, L.D. and Lifshitz, E.M. (1980) Statistical Physics, Part I, Third Edition, Pergamon, Oxford.

[28] Volkov, A.M., Izmest'ev, A.A. and Skrotskii, G.V. (1971) Sov. Phys. JETP, 32, 686.

[29] Marko, H. (1965) Kybernetik, 2, 274.

[30] Bekenstein, J.D. and Mayo, A.E. (2001) preprint gr-qc/0105055.

[31] Bekenstein, J.D. (1981) Phys. Rev. Letters, 46, 623.

[32] Bekenstein, J.D. (1988) Phys. Rev. A, 37, 3434.

[33] Bekenstein, J.D. (1974) Lett. Nuovo Cimento, 11, 467.

[34] Bekenstein, J.D. (1996) in da Silva, A. J. et. al (eds.), XVII Brazilian National Meeting on Particles and Fields, Brazilian Physical Society, São Paulo.

[35] Bekenstein, J.D. (1998) in Proceedings of the VIII Marcel Grossmann Meeting on General Relativity, Piran, T. and Ruffini, R. eds, World Scientific, Singapore, gr-qc/9710076. 
[36] Bekenstein, J.D. (1999) in Novello, M. (ed.), IX Brazilian School of Cosmology and Gravitation, Atlantiscience, Paris, gr-qc/9808028.

[37] Born, M. (1969) Atomic Physics, Eight Edition, Blackie, London.

[38] Penrose, R. and Floyd, R.M. (1971) Nature, 229, 177.

[39] Christodoulou, D. (1970) Phys. Rev. Letters, 25, 1596

[40] Hawking, S.W. (1971) Phys. Rev. Letters, 26, 1344.

[41] Christodoulou, D. and and Ruffini, R. (1971) Phys. Rev. D, 4, 3552.

[42] Bekenstein, J.D. (1998) in Iyer, B.R. and Bhawal, B. (eds.), Black Holes, Gravitational Radiation and the Universe, Kluwer, Dordrecht.

[43] Mayo, A.E. (1998), Phys. Rev. D 58, 104007.

[44] Duez, M.W. et. al (1999) Phys. Rev. D, 60, 104024.

[45] Barvinsky, A, Das, S. and Kunstatter, G. (2000) preprint gr-qc/0012066 and (2001) preprint hep-th/0102061.

[46] Gour, G. (2000) Phys. Rev. D, 61, 124007.

[47] Merzbacher E., 1970, Quantum Mechanics., Second Edition, Wiley, New York.

[48] Bekenstein, J.D. (2002) in Duff, M. and Liu, J. T. (eds.), 2001: A Spacetime Odyssey, World Scientific Publishing, Singapore, hep-th/0107045.

[49] Schiffer, M. (1989) "Black hole spectroscopy", preprint IFT/P-38/89, São Paulo; Peleg, Y. (1995) Phys. Lett. B, 356, 462; Kastrup, H. (1996) Phys. Lett. B, 385, 75; Louko, J. and Mäkelä, J. (1996) Phys. Rev. D, 54, 4982; Barvinsky, A. and Kunstatter, G. (1996) Phys. Lett. B, 329, 231; Berezin, V.A. (1997) Phys. Rev. D, 55, 2139; Vaz, C. and Witten, L. (1999) Phys. Rev. D, 60, 024009; Vaz, C. (2000) Phys. Rev. D, 61064017.

[50] Dolgov, A.D. and Khriplovich, I B. (1997) Phys. Lett. B, 400, 12; Berezin, V.A. (1997) Phys. Rev. D, 55, 2139; Berezin, V.A., Boyarsky, A.M. and Neronov, A.Yu. (1998) Phys. Rev. D, 571118.

[51] Mäkelä, J. and Repo, P. 1997 Phys. Rev. D, 57, 4899.

[52] Mäkelä, J., Repo, P., Luomajoki, M. and Piilonen, J. (2000) Phys. Rev. D, 64, 024018 (2001)

[53] Vaz, C. and Witten, L. (2001) Phys. Rev. D, 63, 024008.

[54] Mukhanov, V.F. (1986) JETP Letters, 44, 63.

[55] Bekenstein, J.D. and Mukhanov, V.F. (1995) Phys. Lett. B, 360, 7. 